\title{
Bi-abundance Photoionization Models for Planetary Nebulae
}

\author{
D. Péquignot ${ }^{1}$, X.-W. Liu ${ }^{2}$, M. J. Barlow ${ }^{2}$, P. J. Storey ${ }^{2}$, C. Morisset $^{3}$ \\ ${ }^{1}$ Observatoire de Paris - Meudon, F-92195 Meudon Cedex, France \\ ${ }^{2}$ Department of Physics \& Astronomy, University College London, \\ Gower Street, London, WC1E 6BT, U.K. \\ ${ }^{3}$ Institut d'Astrophysique de Marseille, Traverse du Siphon, BP 8, \\ F-13376 Marseille Cedex 12, France
}

A new class of planetary nebulae, in which abundances derived from optical recombination lines (ORL) seem to be much larger than those derived from collisionally excited lines (CEL) by up to one or two orders of magnitude is now well identified (Liu, these proceedings and references therein).

Photoionization models including two components, one highly enriched in $\mathrm{C}, \mathrm{N}, \mathrm{O}, \mathrm{Ne}$ and deficient in $\mathrm{H}$ and the other one of more usual composition, can account for most of the numerous spectroscopic data available from UV to far-IR in two of the best observed, most extreme examples, namely NGC 6153 and M142 (Péquignot et al., 2002, and in preparation). The few discrepancies left can generally be understood in terms of inaccuracy of the observations (calibration of infrared line fluxes relative to the optical) and some atomic data, particularly in the unusual conditions prevailing in the H-deficient component.

The H-deficient component, cooled down to very low temperatures by finestructure line (FSL) emission, emits only a small fraction of $\mathrm{H} \beta$, but this is sufficient to make the average temperature derived from the Balmer jump (due to both $\mathrm{H}$ I and He II emissions, with the latter weighted toward the cool gas) to be much smaller than the one derived from the [O III] line ratio, in accordance with observation. The ORLs of $\mathrm{C}, \mathrm{N}, \mathrm{O}, \mathrm{Ne}$ arise essentially from the H-deficient gas, whereas the optical CELs arise from the normal gas, except for possible recombination contributions. Some consequences of this model are as follows.

The predicted intensity of the FSLs is generally not much enhanced with respect to the one obtained in usual homogeneous models, due to the moderate emission measure of the $\mathrm{H}$-deficient component. Also, some FSLs can be partially quenched in this component due to either the very low temperature (e.g., [Ne III], [Ne sc ii]) or the relatively large density (e.g., [O III]).

A large density must prevail in the cool component if the gas pressure is about the same in both components, as expected if they are intimately mixed. This happens to be the case in NGC 6153 , but not in M 1-42, suggesting that these two nebulae are not exactly in the same stage of their evolution. However, in both nebulae, the H-deficient component must be concentrated in the highexcitation region, suggesting that it corresponds to material lately ejected from 
the central region. This trend is detected in NGC 6153 (Liu et al., 2000) and is clearly seen in NGC 6720 (Garnett \& Dinerstein, 2001).

Since all of the optical [O III] emission and most of the $\mathrm{H} \beta$ emission arise from the normal "hot" component, it is expected that the [O III] and $\mathrm{H} \beta$ maps will roughly coincide. This is the case for NGC 6153 , which looks a bona fide $\mathrm{PN}$ in this respect (Liu et al., 2000). Also the average temperature and the temperature fluctuations, as depicted from optical [O III] line ratio maps, will be as predicted by common photoionization models, that is, moderate, with a shallow maximum in the inner regions where some $\mathrm{O}^{2+}$ survive in the hotter $\mathrm{He}^{2+}$ zone. This is again what observations of NGC 6153 reveal.

Doubts, that were expressed about the relevance of pure (static) photoionization models to account for the spectrum of $\mathrm{PNe}$, particularly those with a large difference between their [O III] and Balmer jump temperatures, are no more justified. This diagnostic-temperature difference, relatively small in most nebulae, was very early attributed to temperature fluctuations around an average value (the $t^{2}$ concept of Peimbert, 1967). This empirical concept, useful to improve abundance determinations in homogeneous nebulae, is irrelevant in the situation described by the bi-abundance model since the formal average temperature (close to the average Balmer jump temperature) does not correspond to any parcel of gas. This is in fact the only situation in which the $t^{2}$ approach, made almost unfalsifiable by its mathematical generality, is fundamentally (not only technically) inapplicable. Ironically, it may be that nature has managed to generate such a situation. Inasmuch as the observed $T_{\mathrm{e}}([\mathrm{O} \mathrm{III}])$ fluctuations are not in excess of what usual photoionization model nebulae predict, it must be concluded that little material with intermediate composition and temperature is seen in the PNe so far studied. Thus, the bi-abundance model turns out to be surprisingly successful, suggesting that two totally disconnected episods of mass ejection have taken place.

The mass of $\mathrm{H}$-deficient material is small in the bi-abundance model and the average abundances are relatively close to those of the "normal" component. This result is strongly at variance with the conclusion that could be drawn from applying the $t^{2}$ approach, which necessarily leads to extremely large average abundances for $\mathrm{CNONe}$ in these PNe. Thus the recombination lines do not reflect faithfully the actual average abundance ratios. In particular the helium abundance, very large in some of these $\mathrm{PNe}$ according to conventional analyses, is currently left undetermined, unless one can rely on partially collisionally excited He $i$ lines, such as $\mathrm{He} i 10830 \AA$, whose emission is weighted toward the hot component. Although observational uncertainties are substancial, models favour a moderate abundance for helium in the normal component of both PNe and certainly allows to exclude the homogeneous model. Fluctuations of composition within planetary nebulae can lead to overestimating the helium abundance.

\section{References}

Garnett, D. R., Dinerstein, H. L., 2001, ApJ, 558, 145

Liu, X.-W., Storey, P. J. ,Barlow, M. J., et al. 2000, MNRAS, 312, 585

Peimbert, M., 1967, ApJ, 150, 825

Pé quignot et al., 2002, Rev. Mex. Astron. Astrofis., Ser de Conferencias, 12, 142 